\title{
Clinical Case: Casual Finding of Part of an Intubation Guide after its Use for Exchange and Placement of Double Light Tube
}

\author{
Sepúlveda Haro E, Romero Molina S, Molina Ruiz JC, Bueno García MS, Gil Morata E, Fontaneda \\ Heredia A, Baena Lopez M, Raigón Ponferrada A, Ramirez Aliaga M, Escalona Belmonte JJ, Guerrero \\ Orriach JL* and Cruz Mañas J
}

Department of Anaesthesiology, Virgen de la Victoria University Hospital, Malaga, Spain

A difficult airway is defined as a clinical situation in which a trained anesthesiologist has difficulties to ventilate a patient with a face mask, difficulties for endotracheal intubation, or both. Intubation with a double lumen tube (DLT) may be more difficult than an equivalent intubation with a single-lumen tracheal tube because of its greater external diameter, stiffness and concavity, so its use is not recommended as a technique of initiation in patients with VAD [1].

Rubber elastic gum introducers are established as an aid for intubation. They are mainly indicated in cases of difficulty in intubation by bad laringoscopic vision. Most of the algorithms contemplate the use of this type of guides, because it is demonstrated its efficacy in situations of difficult intubation.

Frova $^{\circledR}$, is a device used as a guide, which allows oxygenation by continuous flow, spontaneous ventilation with capnography wave or assisted ventilation.

Partial obstructions of an endotracheal tube after successful intubation by foreign bodies from the equipment have been described before. We report a case that demonstrates a problem with one of these bugs, the Frova intubation introducer used to help insert a DLT [2-7].

\section{Clinical Case}

A 69-year-old woman with a history of ischemic heart disease, scheduled for aortocoronary bypass surgery of the internal mammary artery to the anterior descending artery by right anterior minithoracotomy without extracorporeal circulation.

After monitoring and anesthetic induction, direct laryngoscopy was performed, observing the larynx with a grade IIB of Cormack-Lehane with the BURP maneuver, and intubation was performed with a Rüsch orotracheal tube with a single light of number 8 , without needing guidance. The correct position is checked by chest inspection of the ventilation, auscultation, capnography curve and airway pressure, and the patient is ventilated correctly. For the placement of the double lumen tube, necessary for the pulmonary block during this type of intervention, the orotracheal tube is exchanged for a left double lumen tube of $37 \mathrm{~F}$, using a Frova introducer as a guide (Cook Medical, Bjaeverskov, Denmark) without incidents.

Subsequently, the position of the TDL is checked by means of fibroscopic visualization. Although the normality of the TDL is verified, a millimeter-shaped, filiform, bluish foreign body, located on the anterior side of the left bronchus light $1 \mathrm{~cm}$ from the tracheal carina, is evident (Figure 1). Its extraction is attempted by fibroscopic aspiration, without success. The use of fiberoptic bronchoscopy forceps is necessary for its removal, so due to the need to use a larger diameter fiberoptic bronchoscope, the change to an orotracheal tube of $8 \mathrm{~mm}$ in diameter was required. The foreign body was extracted with forceps by means of fibroscopic visualization, verifying that it corresponded to a millimeter piece of the Frova introducer detached during its use as an orotracheal tube exchanger.

After the incident, a bronchial blocker was placed through the orotracheal tube and a block of the left lung was performed. The rest of the intervention proceeded without incident, and was subsequently discharged from the Intensive Care Unit and the hospital without any respiratory or cardiological complications.

\section{Discussion}

The need for pulmonary blockade in certain interventions in which the anesthesiologist encounters difficulties with double-lumen tube blockage due to problems in intubation, making a tube exchange through a guide, is one of the possibilities described in the literature, as happened in our case [1-3].

*Corresponding author: Jose Luis Guerrero Orriach, Hospital Virgen de la Victoria, University Campus Teatinos, C.P. 29010, Malaga, Spain

Accepted: September 10, 2019

Published online: September 12, 2019

Citation: Sepúlveda HE, Romero MS, Molina RJC, et al. (2019) Clinical Case: Casual Finding of Part of an Intubation Guide after its Use for Exchange and Placement of Double Light Tube. Arch Fam Med Gen Pract 4(1):90-91 


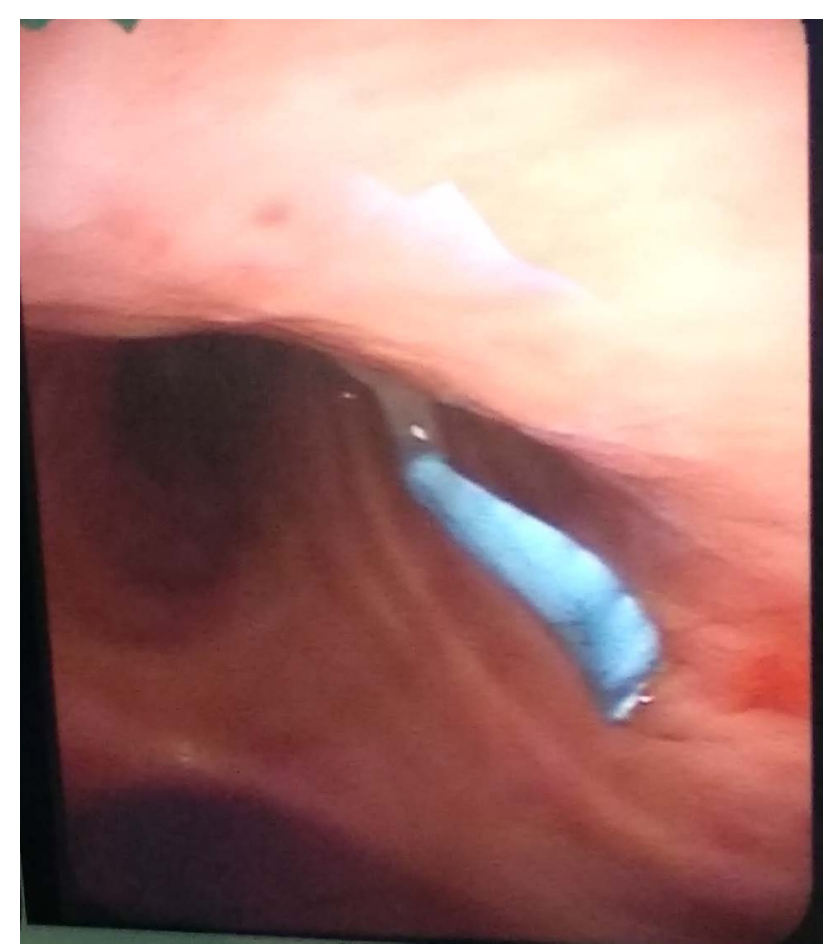

Figure 1: The image shows the right main bronchus and a piece of blue bronchial guide on the bronchial surface, which was removed through the fiberoptic bronchoscope.

In our case, the guide used, although by diameter, gave the possibility of performing the same; it produced one of the complications associated to its use, although nevertheless it was not the axial breakage of the same one; something that would have been evidenced in his withdrawal, but the loss due to laminar rupture, which he did as a key piece in the diagnosis and treatment of fiberoptic bronchoscopy, since it went unnoticed in the inspection of the same after its use [37].

That this type of situation occurs during intubations with an orotracheal tube of a light has a lower incidence, although the difficulty that would have arisen in our case was that, being a sheet of the guide and not a complete part of it, it could be gone unnoticed if the verification technique of the standard DLT were not fiberoptic bronchoscopy.

Therefore, in the perioperative period of patients requiring the use of the frova guidewire for intubation, there are respiratory problems that may be related to this type of complication, they would require imaging or fiberoptic bronchoscopy to rule it out, in addition to checking integrity of the guide whenever it is used.

\section{References}

1. Granell M, Parra MJ, Jiménez MJ, et al. (2018) Revisión del manejo de la vía aérea díficil en cirugía torácica. Revista Española De Anestesiología Y Reanimación 65: 31-40.

2. Kemp JM, Sutton DN (2009) A close shave. Anaesthesia 64: 219220.

3. Michlig SA (2016) Incidental finding of a tracheal fragment from a Frova intubating catheter. Anaesthesia 71: 1492.

4. Mohnssen S, Greggs D (1993) latrogenic aspiration of components of respiratory care equipment. Chest 103: 964-965.

5. Piepho T (2018) Anticipated difficult airway and need of a double lumen tube. Trends In Anaesthesia And Critical Care 18: 37.

6. Schober P, Loer SA, Schwarte LA (2016) Airway obstruction by an unexpected equipment damage. J Clin Anesth 32: 59-61.

7. Vlachtsis $H$, Veltman $M$ (2006) Shearing of a Frova intubating introducer by a bronchocath double lumen tube. Anaesthesia 61: 197-198.

DOI: $10.36959 / 577 / 488$ 